\title{
Multiscale modelling for composites with energetic interfaces at the micro- or nanoscale
}

2014, Vol XX(X) I-16

(C)The Author(s) 2013

Reprints and permissions:

sagepub.co.uk/journalsPermissions.nav DOI: $|0.1| 77 / 108|2865| 35|6| 22$ mms.sagepub.com

\author{
G Chatzigeorgiou, A Javili and P Steinmann \\ Chair of Applied Mechanics, University of Erlangen-Nuremberg, Erlangen, Germany
}

Received I8 September 2013; accepted I8 November 2013

\begin{abstract}
A homogenization framework is developed that accounts for the effect of size at the micro- or nanoscale. This is achieved by endowing the interfaces of the micro- or nanoscopic features with their own independent structure, using the theory of surface elasticity. Following a standard small-strain approach for the microscopic deformation in terms of the macroscopic strain tensor, a Hill-type averaging condition is used to link the two scales. A procedure for determining overall effective properties in the case of composites with elastic components and elastic material interfaces is presented. A special example of multilayered composites demonstrates the correlation between a material interface and a very thin interphase layer.
\end{abstract}

\section{Keywords}

Homogenization, surface elasticity, size effects, nanomaterials.

\section{Introduction}

The surfaces of solid bodies and the interfaces in composites typically exhibit properties different from those of the bulk materials. These differences, caused by processes such as surface oxidation, ageing, coating, atomic rearrangement and the termination of atomic bonds, are present in comparatively thin boundary layers. Surface and interface effects are especially significant for micro- and nanomaterials due to their large surface-area-tovolume ratio.

Surface and interface effects can be modelled using various continuum approaches. Phenomenological models that include surfaces with their own structure date back to the pioneering work of Gibbs [1]. More recent key contributions include those by Gurtin and Murdoch [2] who described surface effects using tensorial surface stresses. Moeckel [3] developed an alternative approach for a moving thermomechanical interface (essentially a two-sided surface). Somewhat later, Daher and Maugin [4] invoked the method of virtual power to endow the surface with its own thermodynamic constituents while dell'Isola and Romano [5] developed a general balance law for continua with interfaces. Based on potential energies Steinmann [6] identified the balance laws for deformational and configurational mechanics.

Homogenization, as pioneered by Hill [7] and Hill and Rice [8], provides a consistent methodology to link the macroscopic and microscopic scales and forms the basis for computational micro-to-macro transitions. A review of different multi-scale approaches can be found in $[9,10]$. The effective mechanical behaviour

\section{Corresponding author:}

A Javili, Chair of Applied Mechanics, University of Erlangen-Nuremberg, Egerlandstr. 5, 91058 Erlangen, Germany.

Email: ali.javili@Itm.uni-erlangen.de

Dedicated to Professor Antonio Di Carlo in recognition of his academic activity 
of composites has been studied thoroughly by various researchers for general and periodic microstructures [7, 11-15]. A computational scheme for homogenization of composites with nonlinear constituents has been presented by Suquet [16]. Homogenization of composites at finite strains has been considered by many authors [17-23].

Motivated by the non-classical behaviour of continua at the nanoscale, the objective of this contribution is to present a novel theoretical micro-to-macro transition framework for problems where the microstructure possesses interface structure. The contribution of the energetic interface to the overall strength of the microscopic representative volume element (RVE) depends on two relative microscopic length scales. (The label 'energetic' denotes that the interface possesses mechanical and constitutive structures. These structures are independent from those of the bulk.) The first is the ratio of the volume of the RVE to the area of the energetic interface. The second is the ratio of the microscopic Helmholtz energies of the interface and the bulk.

The macroscopic and microscopic problems satisfy the assumption of scale separation. Nonetheless, unlike the case where the microstructure contains no energetic interfaces, the magnitude of the relative size of the microscopic problem compared to the macroscale is important when energetic interfaces are considered. The energetic interface structure allows one to capture the increase of specimen strength with decreasing size. Standard micro-to-macro transition frameworks, that is, where the macro- and microstructures possess no enhanced continuum description, cannot capture this strengthening effect. This phenomenon has been investigated numerically using surface elasticity theory (see e.g. [24-27]). The strengthening effect due to the presence of energetic surfaces/interfaces has been studied recently using micromechanics approaches by [28-30]. In those cases, though, the energetic surface has specific geometry (spherical or cylindrical).

This manuscript is organized as follows: the notation and certain key concepts are briefly introduced. The small-strain formulation governing the response of the micro- and macrostructure and the connection between them through volume averaging over the RVE is summarized in Section 2. Appropriate boundary conditions in the RVE that link the microscopic and macroscopic mechanical work are identified using a Hill-type lemma in Section 3. In the same section the governing microscale equations based on the Hill-Mandel criterion are identified for periodic boundary conditions. An analytical procedure for computing the effective stiffness tensor for periodic elastic media with elastic material interfaces is presented in Section 4. The proposed methodology is demonstrated in Section 5 with a particular analytical example of a multilayered composite. The paper closes with a summary and the conclusions of this work.

\section{I.I. Notation}

Direct notation is adopted throughout. Occasional use is made of index notation, the summation convention for repeated indices being implied. Bold letters are used to denote vectors and tensors in order to distinguish them from scalars. In the following, $\delta_{i j}$ is the Kronecker delta and $\boldsymbol{i}$ denotes the second-order identity tensor $[\boldsymbol{i}]_{i j}=\delta_{i j}$. We identify the three forms of single contraction of an $(n+1)$ th-order tensor $\boldsymbol{A}$ and an $(m+1)$ th-order tensor $\boldsymbol{B}$ as the $(n+m)$ th-order tensors $\boldsymbol{A} \cdot \boldsymbol{B}, \boldsymbol{A} \sim \boldsymbol{B}$ and $\boldsymbol{A}_{\sim} \cdot \boldsymbol{B}$, given by

$$
\begin{aligned}
{[\boldsymbol{A} \cdot \boldsymbol{B}} & ]_{i_{1} i_{2} \ldots i_{n+m}}=[\boldsymbol{A}]_{i_{1} i_{2} \ldots i_{n} q}[\boldsymbol{B}]_{q i_{n+1} i_{n+2} \ldots i_{n+m}}, \\
{[\boldsymbol{A} \sim \boldsymbol{B}]_{i_{1} i_{2} \ldots i_{n+m}} } & =[\boldsymbol{A}]_{i_{1} i_{2} \ldots i_{n} q}[\boldsymbol{B}]_{i_{n+1} i_{n+2} \ldots i_{n+m} q}
\end{aligned}
$$

and

$$
[\boldsymbol{A} \underset{\sim}{*} \boldsymbol{B}]_{i_{1} i_{2} \ldots i_{n+m}}=[\boldsymbol{A}]_{q i_{1} i_{2} \ldots i_{n}}[\boldsymbol{B}]_{q i_{n+1} i_{n+2} \ldots i_{n+m}}
$$

respectively. We identify the three forms of the double contraction of an $(n+2)$ th-order tensor $\boldsymbol{A}$ and an $(m+$ 2)th-order tensor $\boldsymbol{B}$ as the $(n+m)$ th-order tensors $\boldsymbol{A}: \boldsymbol{B}, \boldsymbol{A} \tilde{:} \boldsymbol{B}$ and $\boldsymbol{A}: \boldsymbol{B}$, given by

$$
\begin{aligned}
{[\boldsymbol{A}: \boldsymbol{B}]_{i_{1} i_{2} \ldots i_{n+m}} } & =[\boldsymbol{A}]_{i_{1} i_{2} \ldots i_{n} p q}[\boldsymbol{B}]_{p q i_{n+1} i_{n+2} \ldots i_{n+m}}, \\
{[\boldsymbol{A} \tilde{:} \boldsymbol{B}]_{i_{1} i_{2} \ldots i_{n+m}} } & =[\boldsymbol{A}]_{i_{1} i_{2} \ldots i_{n} p q}[\boldsymbol{B}]_{i_{n+1} i_{n+2} \ldots i_{n+m} p q}
\end{aligned}
$$

and

$$
[\boldsymbol{A}: \boldsymbol{B}]_{i_{1} i_{2} \ldots i_{n+m}}=[\boldsymbol{A}]_{p q i_{1} i_{2} \ldots i_{n}}[\boldsymbol{B}]_{p q i_{n+1} i_{n+2} \ldots i_{n+m}}
$$

respectively. The tensor product of an $n$ th-order tensor $\boldsymbol{A}$ and an $m$ th-order tensor $\boldsymbol{B}$ is the $(n+m)$ th-order tensor $\boldsymbol{A} \otimes \boldsymbol{B}$ given by

$$
[\boldsymbol{A} \otimes \boldsymbol{B}]_{i_{1} i_{2} \ldots i_{n+m}}=[\boldsymbol{A}]_{i_{1} i_{2} \ldots i_{n}}[\boldsymbol{B}]_{i_{n+1} i_{n+2} \ldots i_{n+m}} .
$$


Table I. Notation and definition of main variables in the microscale bulk used throughout the text. Microscale interface quantities are notated with a hat above the symbol. Macroscale quantities are notated with a bar above the symbol.

\begin{tabular}{llll}
\hline $\mathbf{x}$ & position vector & $\mathbf{V}$ & volume of RVE \\
$\mathbf{S}$ & area of interface & $\mathbf{u}$ & displacement vector \\
$\boldsymbol{\epsilon}$ & strain tensor & $\boldsymbol{\sigma}$ & stress tensor \\
$\mathbf{t}$ & traction vector & $W$ & energy density function \\
$\mathbf{z}$ & displacement fluctuation vector & $\mathbf{U}$ & third-order fluctuation tensor \\
$\mathbf{C}$ & elasticity tensor & $\mathrm{t}$ & thickness of interphase layer \\
\hline
\end{tabular}

Table 2. Space definitions (volumes, surfaces, curves) and normal vectors to boundaries (surfaces, curves) for macroscale body, microscale bulk and microscale interface.

\begin{tabular}{|c|c|c|c|c|c|c|}
\hline & \multicolumn{2}{|c|}{ Macroscale } & \multicolumn{4}{|c|}{ Microscale } \\
\hline & Body & Body boundary & Bulk & Boundary & Interface & Interface boundary \\
\hline Space & $\overline{\mathcal{B}}$ & $\partial \overline{\mathcal{B}}$ & $\mathcal{B}$ & $\partial \mathcal{B}$ & $\mathcal{I}$ & $\partial \mathcal{I}$ \\
\hline Normal vector & & $\bar{n}$ & & $n$ & $\boldsymbol{n}_{S}$ & $\hat{\boldsymbol{n}}_{S}$ \\
\hline
\end{tabular}

Table 3. Summary of notation used for quantities and tensor operators in macroscale, microscale bulk and microscale energetic interface. In macroscale and microscale bulk, $a, b=1,2,3$, while in microscale interface, $\alpha, \beta=1,2$.

\begin{tabular}{|c|c|}
\hline Macroscale & \\
\hline $\begin{array}{l}\overline{\mathbf{g}}_{a}:=\partial_{\bar{X}^{a}} \overline{\mathbf{x}} \quad \delta_{b}^{a}=\overline{\mathbf{g}}^{a} \cdot \overline{\mathbf{g}}_{b} \\
\overline{\mathbf{i}}:=\delta_{b}^{a} \overline{\mathbf{g}}_{a} \otimes \overline{\mathbf{g}}^{b}=\overline{\mathbf{g}}_{a} \otimes \overline{\mathbf{g}}^{a}=\overline{\mathbf{i}}^{\mathrm{t}} \\
\operatorname{grad}\{\bullet\}:=\partial_{\bar{X}^{a}}\{\bullet\} \otimes \overline{\mathbf{g}}^{a}=\partial_{\overline{\mathbf{x}}}\{\bullet\}=\overline{\operatorname{grad}}\{\bullet\} \cdot \overline{\boldsymbol{i}} \\
\overline{\operatorname{div}}\{\bullet\}:=\partial_{\bar{x}^{a}}\{\bullet\} \cdot \overline{\mathbf{g}}^{a}=\overline{\operatorname{grad}}\{\bullet: \overline{\mathbf{i}}\end{array}$ & \\
\hline Microscale (Bulk) & Microscale (Interface) \\
\hline $\begin{array}{l}\mathbf{g}_{a}:=\partial_{x^{a}} \mathbf{x} \quad \delta_{b}^{a}=\mathbf{g}^{a} \cdot \mathbf{g}_{b} \\
\boldsymbol{i}:=\delta_{b}^{a} \mathbf{g}_{a} \otimes \mathbf{g}^{b}=\mathbf{g}_{a} \otimes \mathbf{g}^{a}=\mathbf{i}^{\mathrm{t}} \\
\operatorname{grad}\left\{\bullet \bullet:=\partial_{x^{a}}\{\bullet\} \otimes \mathbf{g}^{a}=\partial_{\mathbf{x}}\{\bullet\}=\operatorname{grad}\{\bullet\} \cdot \boldsymbol{i}\right. \\
\operatorname{div}\{\bullet\}:=\partial_{x^{a}}\{\bullet\} \cdot \mathbf{g}^{a}=\operatorname{grad}\{\bullet:: \boldsymbol{i}\end{array}$ & 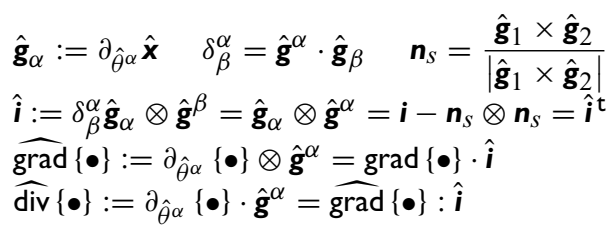 \\
\hline
\end{tabular}

The two non-standard tensor products of two second-order tensors $\boldsymbol{A}$ and $\boldsymbol{B}$ are the fourth-order tensors $[\boldsymbol{A} \bar{\otimes} \boldsymbol{B}]_{i j k l}=[\boldsymbol{A}]_{i k}[\boldsymbol{B}]_{j l}$ and $[\boldsymbol{A} \otimes \boldsymbol{B}]_{i j k l}=[\boldsymbol{A}]_{i l}[\boldsymbol{B}]_{j k}$. The transpose of a second-order tensor $\boldsymbol{A}$, denoted as $\boldsymbol{A}^{\mathrm{t}}$, is a second-order tensor with components $\left[\boldsymbol{A}^{\mathrm{t}}\right]_{i j}=[\boldsymbol{A}]_{j i}$.

Table 1 summarizes the main variables that are used in this work. At the microscale, quantities defined on the interface are differentiated from those on the bulk material by a hat placed above the symbol. That is, $\{\widehat{\bullet}\}$ refers to an interface variable with its bulk counterpart being $\{\bullet\}$. Moreover, macroscopic quantities are differentiated from microscale quantities by an accent placed above the symbol. That is, $\{\boldsymbol{\bullet}\}$ refers to a macroscopic variable with its microscopic counterpart being $\{\bullet\}$. The symbol $\langle\{\bullet\}\rangle$ denotes averaging of the quantity $\{\bullet\}$ over the RVE. Table 2 presents the volumes, surfaces and curves in the microscale used in this paper as well as the vectors connected with them.

We identify as a jump of a quantity $\{\bullet\}$ the relation $\llbracket\{\bullet\} \rrbracket=\{\bullet\}^{+}-\{\bullet\}^{-}$. The Greek letter indices $\alpha$, $\beta, \gamma$, etc. take the values 1 or 2 . The Latin letter indices $a, b, c$, etc. take the values 1,2 or 3 . The general identities that hold in the bulk material and on the interface are presented in Table 3 [6]. In the table $\bar{x}^{a}$ are the Cartesian macro-coordinates, $x^{a}$ are the Cartesian micro-coordinates and $\hat{\theta}^{\alpha}$ denotes the curvilinear microscopic interface coordinates. Moreover, $\overline{\boldsymbol{g}}_{q}$ and $\overline{\boldsymbol{g}}^{q}$ denote Cartesian covariant and contravariant base vectors in the macroscale correspondingly, $\boldsymbol{g}_{q}$ and $\boldsymbol{g}^{q}$ denote Cartesian covariant and contravariant base vectors in the microscopic bulk and $\hat{\boldsymbol{g}}_{\alpha}$ and $\hat{\boldsymbol{g}}^{\alpha}$ denote curvilinear covariant and contravariant base vectors in the microscopic interface respectively. 


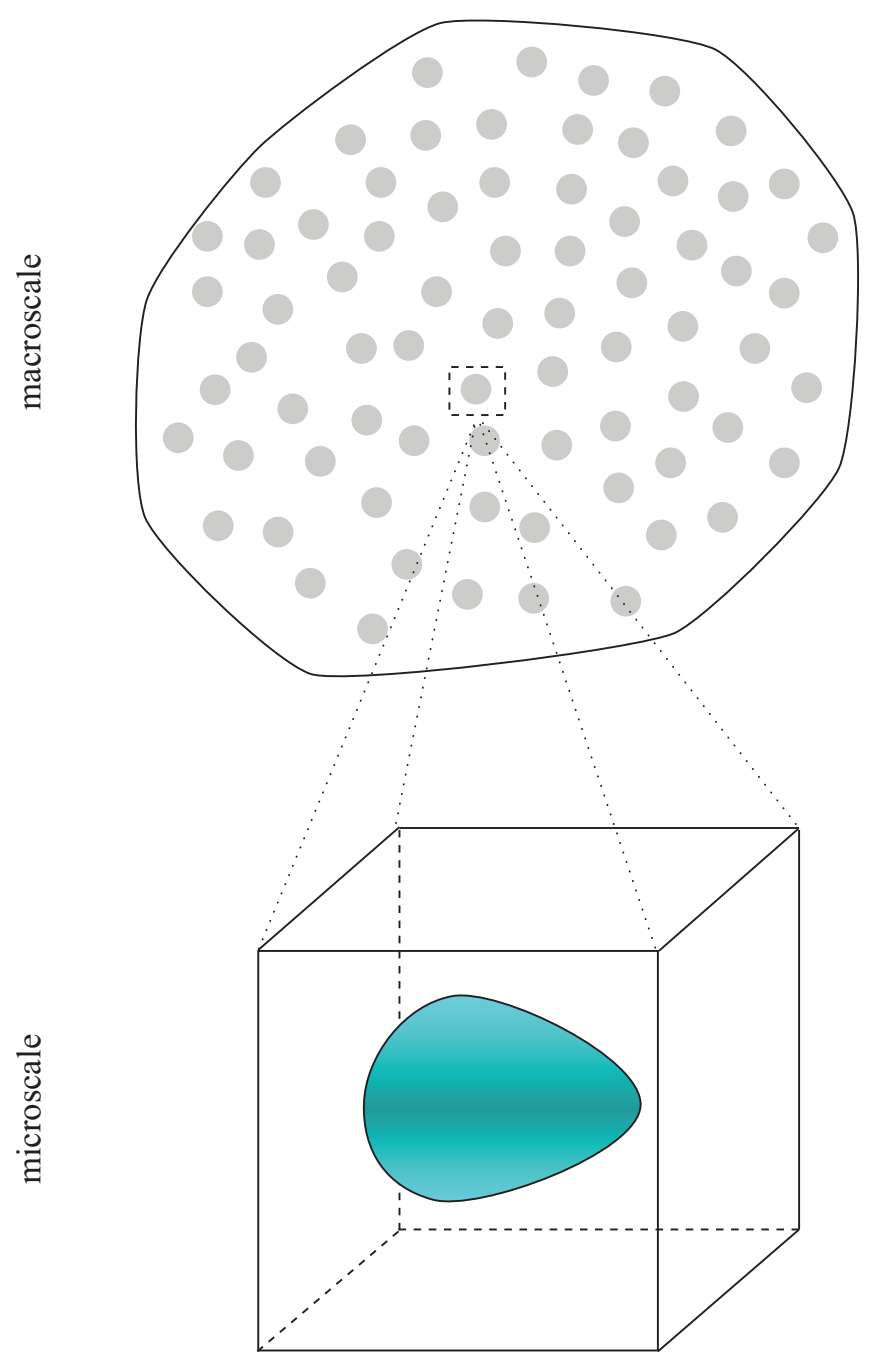

Figure I. Composite material: macroscale and microscale.

\section{Problem definition}

Our intention is to study the behaviour of composites with energetic interfaces subjected to mechanical loading. We can consider two separate scales, the macroscale, which describes the overall body, and the microscale, which describes the RVE of the microstructure (Figure 1). In the subsequent subsections of this section we present the field variables and the main equations that describe the behaviour of the overall body and its microstructure, and we summarize certain key concepts from continuum mechanics. Detailed expositions of continuum mechanics can be found in [31-35], among others. For continuum mechanics of material surfaces, extensive discussion can be found in $[2,6]$.

\section{I. The microscale problem}

At the microscale we consider that the RVE occupies the space $\mathcal{B}$ with volume $V$ and boundary $\partial \mathcal{B}$. The RVE typically consists of two materials, which are separated by a coherent energetic interphase $\mathcal{I}$ (see Figure 2). The interface can either be closed or open intersecting with the boundary $\partial \mathcal{B}$. For the sake of generality we assume throughout the subsequent derivations that the boundary of the interface $\partial \mathcal{I}$ is non-empty space and we discuss the differences that occur when $\partial \mathcal{I}=\emptyset$.

We consider that the energetic interface does not create any discontinuity in the position and displacement, in other words, $\hat{\boldsymbol{x}}=\left.\boldsymbol{x}\right|_{\mathcal{I}}$ and $\hat{\boldsymbol{u}}=\left.\boldsymbol{u}\right|_{\mathcal{I}}$ (kinematically coherent). Moreover, the interface stresses are considered tangential, that is, $\hat{\boldsymbol{\sigma}} \cdot \boldsymbol{n}_{s}=0$. The symmetric microscopic strains in the bulk and the interface are defined by 

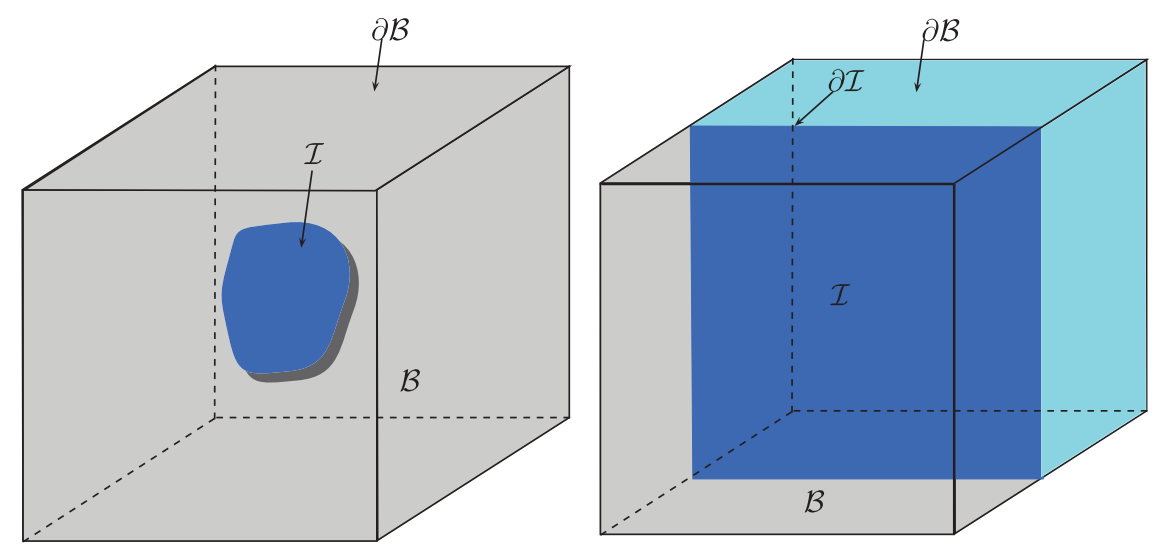

Figure 2. RVEs with closed and open interfaces.

the relations

$$
\boldsymbol{\epsilon}=\frac{1}{2}\left[\boldsymbol{i} \cdot \operatorname{grad} \boldsymbol{u}+[\operatorname{grad} \boldsymbol{u}]^{\mathrm{t}} \cdot \boldsymbol{i}\right] \quad \text { in } \mathcal{B}, \quad \hat{\boldsymbol{\epsilon}}=\frac{1}{2}\left[\hat{\boldsymbol{i}} \cdot \widehat{\operatorname{grad}} \hat{\boldsymbol{u}}+[\widehat{\operatorname{grad}} \hat{\boldsymbol{u}}]^{\mathrm{t}} \cdot \hat{\boldsymbol{i}}\right] \quad \text { on } \mathcal{I} .
$$

We note that the interface strains $\hat{\boldsymbol{\epsilon}}$, as defined in equation $(1)_{2}$, preserve only the tangential components to the interface.

Ignoring micro-body forces in both the bulk and the interface the balance of linear momentum for quasistatic cases leads to [36]

$$
\operatorname{div} \boldsymbol{\sigma}=0 \quad \text { in } \mathcal{B}, \quad \widehat{\operatorname{div}} \hat{\boldsymbol{\sigma}}+\llbracket \boldsymbol{\sigma} \rrbracket \cdot \boldsymbol{n}_{s}=0 \quad \text { on } \mathcal{I} .
$$

In the expressions of the linear momentum the microscopic stresses in both bulk and interface are considered symmetric. The divergence theorems in the bulk and on the interface are written as $[2,6]$

$$
\begin{gathered}
\int_{\partial \mathcal{B}}\{\bullet\} \cdot \boldsymbol{n} \mathrm{d} S=\int_{\mathcal{B}} \operatorname{div}\{\bullet\} \mathrm{d} V+\int_{\mathcal{I}} \llbracket\{\bullet\} \rrbracket \cdot \boldsymbol{n}_{S} \mathrm{~d} S, \\
\int_{\partial \mathcal{I}}\{\bullet\} \cdot \hat{\boldsymbol{n}}_{S} \mathrm{~d} L=\int_{\mathcal{I}} \widehat{\operatorname{div}}\{\bullet\} \mathrm{d} S-\int_{\mathcal{I}} \widehat{\operatorname{div}} \boldsymbol{n}_{S}\{\bullet\} \cdot \boldsymbol{n}_{S} \mathrm{~d} S .
\end{gathered}
$$

Following a similar approach to the one by Gurtin and Murdoch [2] we can then prove the following relations for the bulk and the interface:

$$
\begin{aligned}
& \int_{\partial \mathcal{B}}[\boldsymbol{\sigma} \cdot \boldsymbol{n}] \otimes \boldsymbol{x} \mathrm{d} S=\int_{\mathcal{B}} \operatorname{div} \boldsymbol{\sigma} \otimes \boldsymbol{x} \mathrm{d} V+\int_{\mathcal{I}}\left[\llbracket \boldsymbol{\sigma} \rrbracket \cdot \boldsymbol{n}_{s}\right] \otimes \hat{\boldsymbol{x}} \mathrm{d} S+\int_{\mathcal{B}} \boldsymbol{\sigma} \mathrm{d} V, \\
& \int_{\partial \mathcal{I}}\left[\hat{\boldsymbol{\sigma}} \cdot \hat{\boldsymbol{n}}_{S}\right] \otimes \hat{\boldsymbol{x}} \mathrm{d} L=\int_{\mathcal{I}} \widehat{\operatorname{div}} \hat{\boldsymbol{\sigma}} \otimes \hat{\boldsymbol{x}} \mathrm{d} S+\int_{\mathcal{I}} \hat{\boldsymbol{\sigma}} \mathrm{d} S .
\end{aligned}
$$

Proof. For the first expression we multiply the left-hand side by an arbitrary constant vector $\boldsymbol{k}$. Then using equation (3) $)_{1}$ we get

$$
\begin{aligned}
{\left[\int_{\partial \mathcal{B}}[\boldsymbol{\sigma} \cdot \boldsymbol{n}] \otimes \boldsymbol{x} \mathrm{d} S\right] \cdot \boldsymbol{k} } & =\int_{\partial \mathcal{B}}[\boldsymbol{x} \cdot \boldsymbol{k}] \boldsymbol{\sigma} \cdot \boldsymbol{n} \mathrm{d} S=\int_{\mathcal{B}} \operatorname{div}([\boldsymbol{x} \cdot \boldsymbol{k}] \boldsymbol{\sigma}) \mathrm{d} V+\int_{\mathcal{I}} \llbracket[\hat{\boldsymbol{x}} \cdot \boldsymbol{k}] \boldsymbol{\sigma} \rrbracket \cdot \boldsymbol{n}_{S} \mathrm{~d} S \\
& =\left[\int_{\mathcal{B}} \operatorname{div} \boldsymbol{\sigma} \otimes \boldsymbol{x} \mathrm{d} V+\int_{\mathcal{I}}\left[\llbracket \boldsymbol{\sigma} \rrbracket \cdot \boldsymbol{n}_{S}\right] \otimes \hat{\boldsymbol{x}} \mathrm{d} S+\int_{\mathcal{B}} \boldsymbol{\sigma} \mathrm{d} V\right] \cdot \boldsymbol{k},
\end{aligned}
$$

and the arbitrariness of $\boldsymbol{k}$ completes the proof.

For the second expression we again multiply the left-hand side by an arbitrary constant vector $\boldsymbol{k}$. Then using equation $(3)_{2}$ we get

$$
\begin{aligned}
{\left[\int_{\partial \mathcal{I}}\left[\hat{\boldsymbol{\sigma}} \cdot \hat{\boldsymbol{n}}_{S}\right] \otimes \hat{\boldsymbol{x}} \mathrm{d} L\right] \cdot \boldsymbol{k} } & =\int_{\partial \mathcal{I}}[\hat{\boldsymbol{x}} \cdot \boldsymbol{k}] \hat{\boldsymbol{\sigma}} \cdot \hat{\boldsymbol{n}}_{S} \mathrm{~d} L=\int_{\mathcal{I}} \widehat{\operatorname{div}}([\hat{\boldsymbol{x}} \cdot \boldsymbol{k}] \hat{\boldsymbol{\sigma}}) \mathrm{d} S-\int_{\mathcal{I}} \operatorname{div} \boldsymbol{n}_{S}[\hat{\boldsymbol{x}} \cdot \boldsymbol{k}] \hat{\boldsymbol{\sigma}} \cdot \boldsymbol{n}_{S} \mathrm{~d} S \\
& =\int_{\mathcal{I}}[\widehat{\operatorname{div}} \hat{\boldsymbol{\sigma}} \otimes \hat{\boldsymbol{x}}] \cdot \boldsymbol{k} \mathrm{d} S+\int_{\mathcal{I}} \hat{\boldsymbol{\sigma}} \cdot \hat{\boldsymbol{i}} \cdot \boldsymbol{k} \mathrm{d} S=\left[\int_{\mathcal{I}}[\widehat{\operatorname{div}} \hat{\boldsymbol{\sigma}} \otimes \hat{\boldsymbol{x}}] \mathrm{d} S+\int_{\mathcal{I}} \hat{\boldsymbol{\sigma}} \mathrm{d} S\right] \cdot \boldsymbol{k},
\end{aligned}
$$




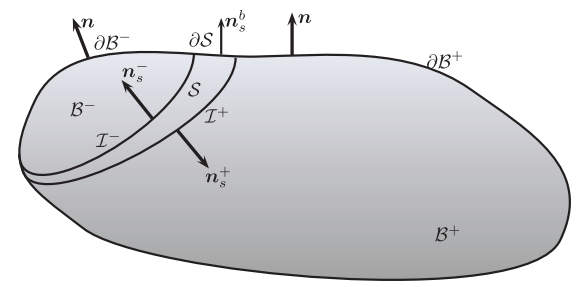

(a)

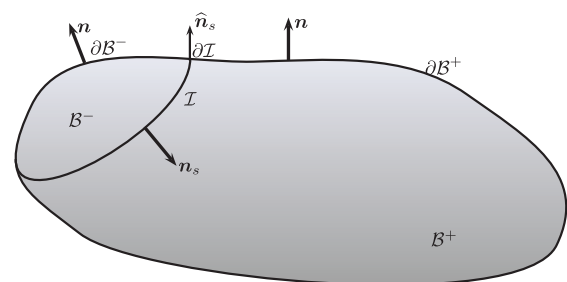

(b)

Figure 3. Body interphase with (a) non-zero thickness and (b) zero thickness.

and the arbitrariness of $\boldsymbol{k}$ completes the proof.

Ignoring scalar-valued, liquid-like interface tension, the constitutive relations that connect the stresses with the small strains for both the bulk and the interface are usually provided through the introduction of appropriate energy density functions. We consider the energy density functions $W(\boldsymbol{\epsilon}), \widehat{W}(\hat{\boldsymbol{\epsilon}})$ such that the bulk and interface stresses are given by

$$
\boldsymbol{\sigma}(\boldsymbol{\epsilon}):=\frac{\partial W}{\partial \boldsymbol{\epsilon}} \quad \text { and } \quad \hat{\boldsymbol{\sigma}}(\hat{\boldsymbol{\epsilon}}):=\frac{\partial \widehat{W}}{\partial \hat{\boldsymbol{\epsilon}}} .
$$

The increments of the energy density functions are then written as

$$
\delta W=\boldsymbol{\sigma}: \delta \boldsymbol{\epsilon}, \quad \delta \widehat{W}=\hat{\boldsymbol{\sigma}}: \delta \hat{\boldsymbol{\epsilon}} .
$$

\subsection{The macroscale problem}

In the macroscale we consider a continuum body that occupies the configuration $\overline{\mathcal{B}}$ with boundary $\partial \overline{\mathcal{B}}$. For static cases, the deformation is characterized by the symmetric macroscopic strain $\overline{\boldsymbol{\epsilon}}$,

$$
\overline{\boldsymbol{\epsilon}}=\frac{1}{2}\left[\overline{\boldsymbol{i}} \cdot \overline{\operatorname{grad}} \overline{\boldsymbol{u}}+[\overline{\operatorname{grad}} \overline{\boldsymbol{u}}]^{\mathrm{t}} \cdot \overline{\boldsymbol{i}}\right] .
$$

In the absence of mechanical body forces, the macroscopic equilibrium equation is written in terms of the symmetric macroscopic stress $\bar{\sigma}$ as

$$
\overline{\operatorname{div}} \overline{\boldsymbol{\sigma}}=0 .
$$

We identify the macroscopic energy density function $\bar{W}$, whose increment $\delta \bar{W}$ is given by the relation

$$
\delta \bar{W}=\overline{\boldsymbol{\sigma}}: \delta \overline{\boldsymbol{\epsilon}} .
$$

In micromechanics it is common to connect macroscopic and microscopic scales through volume averaging of microscopic quantities over a specific RVE. Using the divergence theorem $(3)_{1}$ and considering that $\boldsymbol{u}$ is continuous across the interface, the volume average of the microscopic strain over the RVE is given by

$$
\langle\boldsymbol{\epsilon}\rangle=\frac{1}{V} \int_{\mathcal{B}} \boldsymbol{\epsilon} \mathrm{d} V=\frac{1}{2 V} \int_{\partial \mathcal{B}}[\boldsymbol{u} \otimes \boldsymbol{n}+\boldsymbol{n} \otimes \boldsymbol{u}] \mathrm{d} S .
$$

Concerning the average stress, the appearance of non-zero stresses on the interface requires accounting for them when averaging over the entire RVE: when the interface is considered to have a small but finite thickness, the entire body in the undeformed configuration is split into three parts, $\mathcal{B}^{+}, \mathcal{B}^{-}$and $\mathcal{S}$ (Figure 3(a)), and the average stress is given by

$$
\langle\boldsymbol{\sigma}\rangle=\frac{1}{V} \int_{\mathcal{B}^{+}} \sigma \mathrm{d} V+\frac{1}{V} \int_{\mathcal{B}^{-}} \sigma \mathrm{d} V+\frac{1}{V} \int_{\mathcal{S}} \sigma \mathrm{d} V
$$


Under no body forces, the divergence theorem $(3)_{1}$ and the equilibrium equation $(2)_{1}$ allow us to write the third integral as

$$
\int_{\mathcal{S}} \boldsymbol{\sigma} \mathrm{d} V=\int_{\mathcal{I}^{+}}\left[\boldsymbol{\sigma}^{+} \cdot \boldsymbol{n}_{S}^{+}\right] \otimes \boldsymbol{x} \mathrm{d} S+\int_{\mathcal{I}^{-}}\left[\boldsymbol{\sigma}^{-} \cdot \boldsymbol{n}_{S}^{-}\right] \otimes \boldsymbol{x} \mathrm{d} S+\int_{\partial \mathcal{S}}\left[\boldsymbol{\sigma} \cdot \boldsymbol{n}_{S}^{b}\right] \otimes \boldsymbol{x} \mathrm{d} S,
$$

where $\mathcal{I}^{+}$and $\mathcal{I}^{-}$are the boundaries of the interface inside the body and $\partial \mathcal{S}$ denotes the boundaries of the interface on the boundary of the body, while $\boldsymbol{n}_{s}^{+}, \boldsymbol{n}_{s}^{-}$and $\boldsymbol{n}_{s}^{b}$ are the corresponding normal unit vectors to these interfaces (see Figure 3(a)). In the limit case where the interface has zero thickness,

$$
\partial \mathcal{S} \rightarrow \partial \mathcal{I}, \quad \mathcal{I}^{+} \rightarrow \mathcal{I}^{-} \rightarrow \mathcal{I}, \quad \boldsymbol{n}_{s}^{+}=-\boldsymbol{n}_{s}^{-}=\boldsymbol{n}_{s},\left.\quad \boldsymbol{x}\right|_{\mathcal{I}}=\hat{\boldsymbol{x}}, \quad\left[\boldsymbol{\sigma} \cdot \boldsymbol{n}_{s}^{b}\right] \otimes \boldsymbol{x} \rightarrow\left[\hat{\boldsymbol{\sigma}} \cdot \hat{\boldsymbol{n}}_{s}\right] \otimes \hat{\boldsymbol{x}},
$$

and equation (11) is rewritten as

$$
\langle\boldsymbol{\sigma}\rangle=\frac{1}{V} \int_{\mathcal{B}} \boldsymbol{\sigma} \mathrm{d} V+\frac{1}{V} \int_{\mathcal{I}}\left[\llbracket \boldsymbol{\sigma} \rrbracket \cdot \boldsymbol{n}_{s}\right] \otimes \hat{\boldsymbol{x}} \mathrm{d} S+\frac{1}{V} \int_{\partial \mathcal{I}}\left[\hat{\boldsymbol{\sigma}} \cdot \hat{\boldsymbol{n}}_{s}\right] \otimes \hat{\boldsymbol{x}} \mathrm{d} L .
$$

Using equations (2) $)_{1,2}$ and (4) $)_{1,2}$, the volume average of the microscopic stress over the RVE can be expressed in the two alternative forms

$$
\langle\boldsymbol{\sigma}\rangle=\frac{1}{V} \int_{\mathcal{B}} \boldsymbol{\sigma} \mathrm{d} V+\frac{1}{V} \int_{\mathcal{I}} \hat{\boldsymbol{\sigma}} \mathrm{d} S=\frac{1}{V} \int_{\partial \mathcal{B}} \boldsymbol{t} \otimes \boldsymbol{x} \mathrm{d} S+\frac{1}{V} \int_{\partial \mathcal{I}} \hat{\boldsymbol{t}} \otimes \hat{\boldsymbol{x}} \mathrm{d} L,
$$

where $\boldsymbol{t}:=\boldsymbol{\sigma} \cdot \boldsymbol{n}$ and $\hat{\boldsymbol{t}}:=\hat{\boldsymbol{\sigma}} \cdot \hat{\boldsymbol{n}}_{s}$ are the traction vectors in the bulk and on the interface respectively. As we observe, the average stress differs from the average stress of bodies with non-energetic interfaces that cause traction jump conditions [37], since on the second (boundary) form of equation (12) there is an explicit contribution of the interface stresses through the integral over the boundary curve $\partial \mathcal{I}$. Duan et al. [28] have utilized the definition of Benveniste and Miloh [37] for the macroscopic stress, which holds in the case of spherical inclusions since the interface is closed and $\partial \mathcal{I}=\emptyset$. This definition, though, is not correct when the interface is open.

In a similar manner, the average energy density function increment $\langle\delta W\rangle=\langle\boldsymbol{\sigma}: \delta \boldsymbol{\epsilon}\rangle$ includes the interface counterpart and it is given by the expression

$$
\langle\delta W\rangle=\frac{1}{V} \int_{\mathcal{B}} \boldsymbol{\sigma}: \delta \boldsymbol{\epsilon} \mathrm{d} V+\frac{1}{V} \int_{\mathcal{I}} \hat{\boldsymbol{\sigma}}: \delta \hat{\boldsymbol{\epsilon}} \mathrm{d} S=\frac{1}{V} \int_{\partial \mathcal{B}} \boldsymbol{t} \cdot \delta \boldsymbol{u} \mathrm{d} S+\frac{1}{V} \int_{\partial \mathcal{I}} \hat{\boldsymbol{t}} \cdot \delta \hat{\boldsymbol{u}} \mathrm{d} L .
$$

Proof. Assuming that the interface has a small but finite thickness, the average energy density increment over the RVE is written as

$$
\langle\boldsymbol{\sigma}: \delta \boldsymbol{\epsilon}\rangle=\frac{1}{V} \int_{\mathcal{B}^{+}} \boldsymbol{\sigma}: \delta \boldsymbol{\epsilon} \mathrm{d} V+\frac{1}{V} \int_{\mathcal{B}^{-}} \sigma: \delta \epsilon \mathrm{d} V+\frac{1}{V} \int_{\mathcal{S}} \sigma: \delta \boldsymbol{\epsilon} V .
$$

Under no body forces, equation $(1)_{1}$, the divergence theorem $(3)_{1}$ and the equilibrium equation $(2)_{1}$ allow us to write the third integral as

$$
\begin{aligned}
\int_{\mathcal{S}} \boldsymbol{\sigma}: \delta \boldsymbol{\epsilon} \mathrm{d} V & =\frac{1}{V} \int_{\mathcal{S}} \operatorname{div}(\delta \boldsymbol{u} \cdot \boldsymbol{\sigma}) \mathrm{d} V \\
& =\int_{\mathcal{I}^{+}} \delta \boldsymbol{u} \cdot \boldsymbol{\sigma}^{+} \cdot \boldsymbol{n}_{s}^{+} \mathrm{d} S+\int_{\mathcal{I}^{-}} \delta \boldsymbol{u} \cdot \boldsymbol{\sigma}^{-} \cdot \boldsymbol{n}_{S}^{-} \mathrm{d} S+\int_{\partial \mathcal{S}} \delta \boldsymbol{u} \cdot \boldsymbol{\sigma} \cdot \boldsymbol{n}_{s}^{b} \mathrm{~d} S .
\end{aligned}
$$

In the limit case where the interface has zero thickness,

$$
\partial \mathcal{S} \rightarrow \partial \mathcal{I}, \quad \mathcal{I}^{+} \rightarrow \mathcal{I}^{-} \rightarrow \mathcal{I}, \quad \boldsymbol{n}_{s}^{+}=-\boldsymbol{n}_{s}^{-}=\boldsymbol{n}_{s},\left.\quad \delta \boldsymbol{u}\right|_{\mathcal{I}}=\delta \hat{\boldsymbol{u}}, \quad \delta \boldsymbol{u} \cdot \boldsymbol{\sigma} \cdot \boldsymbol{n}_{s}^{b} \rightarrow \delta \hat{\boldsymbol{u}} \cdot \hat{\boldsymbol{\sigma}} \cdot \hat{\boldsymbol{n}}_{s},
$$

we obtain

$$
\langle\boldsymbol{\sigma}: \delta \boldsymbol{\epsilon}\rangle=\frac{1}{V} \int_{\mathcal{B}} \boldsymbol{\sigma}: \delta \boldsymbol{\epsilon} \mathrm{d} V+\frac{1}{V} \int_{\mathcal{I}} \delta \hat{\boldsymbol{u}} \cdot \llbracket \boldsymbol{\sigma} \rrbracket \cdot \boldsymbol{n}_{S} \mathrm{~d} S+\frac{1}{V} \int_{\partial \mathcal{I}} \delta \hat{\boldsymbol{u}} \cdot \hat{\boldsymbol{\sigma}} \cdot \hat{\boldsymbol{n}}_{s} \mathrm{~d} L
$$


With regard to the first expression of equation (13), equations $(1)_{2},(2)_{2},(3)_{2}$ and the relation $\hat{\boldsymbol{\sigma}} \cdot \boldsymbol{n}_{s}=0$ allow us to write (14) as

$$
\begin{aligned}
\langle\boldsymbol{\sigma}: \delta \boldsymbol{\epsilon}\rangle & =\frac{1}{V} \int_{\mathcal{B}} \boldsymbol{\sigma}: \delta \boldsymbol{\epsilon} \mathrm{d} V+\frac{1}{V} \int_{\mathcal{I}} \delta \hat{\boldsymbol{u}} \cdot \llbracket \boldsymbol{\sigma} \rrbracket \cdot \boldsymbol{n}_{S} \mathrm{~d} S+\frac{1}{V} \int_{\mathcal{I}} \widehat{\operatorname{div}}(\delta \hat{\boldsymbol{u}} \cdot \hat{\boldsymbol{\sigma}}) \mathrm{d} S \\
& =\frac{1}{V} \int_{\mathcal{B}} \boldsymbol{\sigma}: \delta \boldsymbol{\epsilon} \mathrm{d} V+\frac{1}{V} \int_{\mathcal{I}} \hat{\boldsymbol{\sigma}}: \delta \hat{\boldsymbol{\epsilon}} \mathrm{d} S .
\end{aligned}
$$

With regard to the second expression of equation (13), equations (2),$(3)_{1}$ and the continuity of $\boldsymbol{u}$ across the interface allow us to write (14) as

$$
\begin{aligned}
\langle\boldsymbol{\sigma}: \delta \boldsymbol{\epsilon}\rangle & =\frac{1}{V} \int_{\mathcal{B}} \operatorname{div}(\delta \boldsymbol{u} \cdot \boldsymbol{\sigma}) \mathrm{d} V+\frac{1}{V} \int_{\mathcal{I}} \llbracket \delta \boldsymbol{u} \cdot \boldsymbol{\sigma} \rrbracket \cdot \boldsymbol{n}_{S} \mathrm{~d} S+\frac{1}{V} \int_{\partial \mathcal{I}} \hat{\boldsymbol{t}} \cdot \delta \hat{\boldsymbol{u}} \mathrm{d} L \\
& =\frac{1}{V} \int_{\partial \mathcal{B}} \boldsymbol{t} \cdot \delta \boldsymbol{u} \mathrm{d} S+\frac{1}{V} \int_{\partial \mathcal{I}} \hat{\boldsymbol{t}} \cdot \delta \hat{\boldsymbol{u}} \mathrm{d} L .
\end{aligned}
$$

Following classical micromechanics arguments the macroscopic stresses and strains are considered equal to the average microscopic stresses and strains respectively over the RVE, as defined in the above:

$$
\bar{\sigma}=\langle\sigma\rangle, \quad \bar{\epsilon}=\langle\boldsymbol{\epsilon}\rangle .
$$

\section{Transition from micro to macro level: Macroscopic fields and RVE problem}

\section{I. Appropriate RVE boundary conditions: Hill's lemma}

The solution of the microscopic problem requires appropriate boundary conditions that will satisfy the HillMandel condition. In this section we want to identify the types of boundary condition (uniform or periodic fields) on the RVE under which the Hill-Mandel condition holds, that is, the average of the increment of the energy density function (13) $)_{2}$ over the volume of the RVE is equal to the macroscopic increment of the energy density function. In order to achieve our goal, we will need to express Hill's lemma considering the interface.

Hill's lemma In terms of the displacement increments and tractions, Hill's lemma is written as

$$
\langle\boldsymbol{\sigma}: \delta \boldsymbol{\epsilon}\rangle-\langle\boldsymbol{\sigma}\rangle:\langle\delta \boldsymbol{\epsilon}\rangle=\frac{1}{V} \int_{\partial \mathcal{B}}[\delta \boldsymbol{u}-\langle\delta \boldsymbol{\epsilon}\rangle \cdot \boldsymbol{x}] \cdot[\boldsymbol{\sigma} \cdot \boldsymbol{n}-\langle\boldsymbol{\sigma}\rangle \cdot \boldsymbol{n}] \mathrm{d} S+\frac{1}{V} \int_{\partial \mathcal{I}}[\delta \hat{\boldsymbol{u}}-\langle\delta \boldsymbol{\epsilon}\rangle \cdot \hat{\boldsymbol{x}}] \cdot\left[\hat{\boldsymbol{\sigma}} \cdot \hat{\boldsymbol{n}}_{S}\right] \mathrm{d} L
$$

which, if we neglect the energetic interface, takes its classical form. Surprisingly, in the above expression the average stress is not present in the second integral that concerns the interface.

Proof. Due to the continuity of the body we have

$$
\frac{1}{V} \int_{\partial \mathcal{B}} \boldsymbol{x} \otimes \boldsymbol{n} \mathrm{d} S=\boldsymbol{i}
$$

Thus, using equation (10) and the symmetry of the stress tensor, we obtain

$$
\begin{aligned}
& \frac{1}{V} \int_{\partial \mathcal{B}}[\delta \boldsymbol{u}-\langle\delta \boldsymbol{\epsilon}\rangle \cdot \boldsymbol{x}] \cdot[\boldsymbol{\sigma} \cdot \boldsymbol{n}-\langle\boldsymbol{\sigma}\rangle \cdot \boldsymbol{n}] \mathrm{d} S=\frac{1}{V} \int_{\partial \mathcal{B}} \boldsymbol{t} \cdot \delta \boldsymbol{u} \mathrm{d} S-\frac{1}{V} \int_{\partial \mathcal{B}} \boldsymbol{t} \otimes \boldsymbol{x} \mathrm{d} S:\langle\delta \boldsymbol{\epsilon}\rangle \\
& -\langle\boldsymbol{\sigma}\rangle: \frac{1}{2 V} \int_{\partial \mathcal{B}}[\delta \boldsymbol{u} \otimes \boldsymbol{n}+\boldsymbol{n} \otimes \delta \boldsymbol{u}] \mathrm{d} S \\
& +[\langle\delta \boldsymbol{\epsilon}\rangle \cdot\langle\boldsymbol{\sigma}\rangle]: \frac{1}{V} \int_{\partial \mathcal{B}} \boldsymbol{x} \otimes \boldsymbol{n} \mathrm{d} S \\
& =\frac{1}{V} \int_{\partial \mathcal{B}} \boldsymbol{t} \cdot \delta \boldsymbol{u} \mathrm{d} S-\frac{1}{V} \int_{\partial \mathcal{B}} \boldsymbol{t} \otimes \boldsymbol{x} \mathrm{d} S:\langle\delta \boldsymbol{\epsilon}\rangle,
\end{aligned}
$$


and

$$
\frac{1}{V} \int_{\partial \mathcal{I}}[\delta \hat{\boldsymbol{u}}-\langle\delta \boldsymbol{\epsilon}\rangle \cdot \hat{\boldsymbol{x}}] \cdot\left[\hat{\boldsymbol{\sigma}} \cdot \hat{\boldsymbol{n}}_{S}\right] \mathrm{d} L=\frac{1}{V} \int_{\partial \mathcal{B}} \hat{\boldsymbol{t}} \cdot \delta \hat{\boldsymbol{u}} \mathrm{d} S-\frac{1}{V} \int_{\partial \mathcal{B}} \hat{\boldsymbol{t}} \otimes \hat{\boldsymbol{x}} \mathrm{d} S:\langle\delta \boldsymbol{\epsilon}\rangle
$$

By adding the two expressions and taking into account equations (12) and (13) we obtain Hill's lemma (15).

The types of boundary condition for which the right-hand side of equation (15) vanishes are the following:

a) Linear displacements

$$
\boldsymbol{u}=\langle\boldsymbol{\epsilon}\rangle \cdot \boldsymbol{x} \text { on } \partial \mathcal{B} \text { and } \hat{\boldsymbol{u}}=\langle\boldsymbol{\epsilon}\rangle \cdot \hat{\boldsymbol{x}} \text { on } \partial \mathcal{I}
$$

b) Uniform tractions

$$
\boldsymbol{t}=\boldsymbol{\sigma} \cdot \boldsymbol{n}=\langle\boldsymbol{\sigma}\rangle \cdot \boldsymbol{n} \text { on } \partial \mathcal{B} \text { and } \hat{\boldsymbol{t}}=\hat{\boldsymbol{\sigma}} \cdot \hat{\boldsymbol{n}}_{s}=0 \text { on } \partial \mathcal{I} .
$$

c) Periodic conditions in periodic microstructure

$$
\boldsymbol{u}=\langle\boldsymbol{\epsilon}\rangle \cdot \boldsymbol{x}+\boldsymbol{z}, \boldsymbol{z} \text { periodic, } \boldsymbol{t} \text { antiperiodic and } \hat{\boldsymbol{u}}=\langle\boldsymbol{\epsilon}\rangle \cdot \hat{\boldsymbol{x}}+\hat{\boldsymbol{z}}, \hat{\boldsymbol{z}} \text { periodic, } \hat{\boldsymbol{t}} \text { antiperiodic. }
$$

For a closed interface $\partial \mathcal{I}=\emptyset$ the integral over $\partial \mathcal{I}$ vanishes. Then the corresponding boundary conditions are:

a) Linear displacements

$$
\boldsymbol{u}=\langle\boldsymbol{\epsilon}\rangle \cdot \boldsymbol{x} \text { on } \partial \mathcal{B}
$$

b) Uniform tractions

$$
\boldsymbol{t}=\boldsymbol{\sigma} \cdot \boldsymbol{n}=\langle\boldsymbol{\sigma}\rangle \cdot \boldsymbol{n} \text { on } \partial \mathcal{B} .
$$

c) Periodic conditions in periodic microstructure

$$
\boldsymbol{u}=\langle\boldsymbol{\epsilon}\rangle \cdot \boldsymbol{x}+\boldsymbol{z}, \boldsymbol{z} \text { periodic, } \boldsymbol{t} \text { antiperiodic on } \partial \mathcal{B} .
$$

\subsection{Periodicity conditions}

In the sequel we focus on periodic boundary conditions and we are going to identify the RVE problem by using the Hill-Mandel condition. We assume that the tractions are antiperiodic and the microscopic displacement $\boldsymbol{u}$ is the sum of a linear and a periodic term,

$$
\boldsymbol{u}=\overline{\boldsymbol{\epsilon}} \cdot \boldsymbol{x}+\boldsymbol{z}, \quad \hat{\boldsymbol{u}}=\overline{\boldsymbol{\epsilon}} \cdot \hat{\boldsymbol{x}}+\hat{\boldsymbol{z}}, \quad \hat{\boldsymbol{z}}=\left.\boldsymbol{z}\right|_{\mathcal{I}},
$$

where $\boldsymbol{z}=\boldsymbol{z}(\boldsymbol{x})$ is a periodic vector-valued function of $\boldsymbol{x}$. In this case equation (10) and the symmetry of the strain tensor lead to $\langle\boldsymbol{\epsilon}\rangle=\overline{\boldsymbol{\epsilon}}$. Moreover, using (12), the average of the increment of the energy density function (13) is written as [AQ: 1]

$$
\begin{aligned}
\langle\delta W\rangle & =\frac{1}{V} \int_{\partial \mathcal{B}} \boldsymbol{t} \cdot \delta \boldsymbol{u} \mathrm{d} S+\frac{1}{V} \int_{\partial \mathcal{I}} \hat{\boldsymbol{t}} \cdot \delta \hat{\boldsymbol{u}} \mathrm{d} L \\
& =\left[\frac{1}{V} \int_{\partial \mathcal{B}} \boldsymbol{t} \otimes \boldsymbol{x} \mathrm{d} S+\frac{1}{V} \int_{\partial \mathcal{I}} \hat{\boldsymbol{t}} \otimes \hat{\boldsymbol{x}} \mathrm{d} L\right]: \delta \overline{\boldsymbol{\epsilon}}+\frac{1}{V} \int_{\partial \mathcal{B}} \boldsymbol{t} \cdot \delta \boldsymbol{z} \mathrm{d} S+\frac{1}{V} \int_{\partial \mathcal{I}} \hat{\boldsymbol{t}} \cdot \delta \hat{\boldsymbol{z}} \mathrm{d} L \\
& =\langle\boldsymbol{\sigma}\rangle: \delta \overline{\boldsymbol{\epsilon}}+\frac{1}{V} \int_{\partial \mathcal{B}} \boldsymbol{t} \cdot \delta \boldsymbol{z} \mathrm{d} S+\frac{1}{V} \int_{\partial \mathcal{I}} \hat{\boldsymbol{t}} \cdot \delta \hat{\boldsymbol{z}} \mathrm{d} L
\end{aligned}
$$

Considering $\langle\boldsymbol{\sigma}\rangle=\overline{\boldsymbol{\sigma}}$ and the Hill-Mandel condition $\langle\delta W\rangle=\delta \bar{W}$, we obtain the RVE problem in the form

$$
\int_{\partial \mathcal{B}} \boldsymbol{t} \cdot \delta \boldsymbol{z} \mathrm{d} S+\int_{\partial \mathcal{I}} \hat{\boldsymbol{t}} \cdot \delta \hat{\boldsymbol{z}} \mathrm{d} L=0
$$

The two integrals vanish due to the periodicity of $\boldsymbol{z}, \hat{\boldsymbol{z}}$ and the antiperiodicity of $\boldsymbol{t}, \hat{\boldsymbol{t}}$. With the help of the divergence theorems (3) 1,2 and the relation $\hat{\boldsymbol{\sigma}} \cdot \boldsymbol{n}_{s}=0$, equation (17) is expressed as

$$
\int_{\mathcal{B}} \operatorname{div}(\delta \boldsymbol{z} \cdot \boldsymbol{\sigma}) \mathrm{d} V+\int_{\mathcal{I}} \widehat{\operatorname{div}}(\delta \hat{\boldsymbol{z}} \cdot \hat{\boldsymbol{\sigma}}) \mathrm{d} S+\int_{\mathcal{I}} \delta \hat{\boldsymbol{z}} \cdot \llbracket \boldsymbol{\sigma} \rrbracket \cdot \boldsymbol{n}_{s} \mathrm{~d} S=0
$$


or, likewise,

$$
\int_{\mathcal{B}} \delta \boldsymbol{z} \cdot \operatorname{div} \boldsymbol{\sigma} \mathrm{d} V+\int_{\mathcal{I}} \delta \hat{\boldsymbol{z}} \cdot\left[\widehat{\operatorname{div}} \hat{\boldsymbol{\sigma}}+\llbracket \boldsymbol{\sigma} \rrbracket \cdot \boldsymbol{n}_{s}\right] \mathrm{d} S+\int_{\mathcal{B}} \boldsymbol{\sigma}: \operatorname{grad} \delta \boldsymbol{z} \mathrm{d} V+\int_{\mathcal{I}} \hat{\boldsymbol{\sigma}}: \widehat{\operatorname{grad}} \delta \hat{\boldsymbol{z}} \mathrm{d} S=0 .
$$

Using the equilibrium equations $(2)_{1,2}$ we obtain the RVE problem in the form

$$
\int_{\mathcal{B}} \sigma: \operatorname{grad} \delta \boldsymbol{z} \mathrm{d} V+\int_{\mathcal{I}} \hat{\boldsymbol{\sigma}}: \widehat{\operatorname{grad}} \delta \hat{\boldsymbol{z}} \mathrm{d} S=0 .
$$

For closed interfaces, the form (18) of the RVE problem is exactly the same, while in the form (17) the second integral vanishes.

\section{Periodic linearly elastic materials with elastic energetic interfaces: Effective properties}

In linear elasticity the constitutive law between stresses and strains for a bulk material is the usual one,

$$
\sigma=C: \epsilon=C: \operatorname{grad} u,
$$

where $\boldsymbol{C}$ is the constant stiffness tensor. For the energetic interface the constitutive law takes a similar form [2],

$$
\hat{\boldsymbol{\sigma}}=\widehat{\boldsymbol{C}}: \hat{\boldsymbol{\epsilon}}=\widehat{\boldsymbol{C}}: \widehat{\operatorname{grad}} \hat{\boldsymbol{u}},
$$

where $\widehat{\boldsymbol{C}}$ is the interface stiffness tensor whose only non-zero components are tangential, since it satisfies the property $(1 / 2) \widehat{\boldsymbol{C}}:[\hat{\boldsymbol{i}} \otimes \hat{\boldsymbol{i}}+\hat{\boldsymbol{i}} \bar{\otimes} \hat{\boldsymbol{i}}]=\widehat{\boldsymbol{C}}$. Both $\boldsymbol{C}$ and $\widehat{\boldsymbol{C}}$ are periodic tensors on $\mathcal{H}$. Introducing the test vector fields $\delta \eta \in \mathcal{H}_{0}^{1}(\mathcal{B}), \delta \hat{\boldsymbol{\eta}} \in \overline{\mathcal{H}}_{0}^{1}(\mathcal{I})$ and using an analogous approach to the one for obtaining equation (18), the virtual work principle is expressed in the form

$$
\int_{\mathcal{B}} \operatorname{grad} \delta \eta: \sigma \mathrm{d} V+\int_{\mathcal{I}} \widehat{\operatorname{grad}} \delta \hat{\eta}: \hat{\sigma} \mathrm{d} S=0 .
$$

Using equations (19), (20) and (16) the virtual work principle is rewritten as

$$
\begin{aligned}
\int_{\mathcal{B}} \operatorname{grad} \delta \eta:[\boldsymbol{C}: \operatorname{grad} \boldsymbol{z}] \mathrm{d} V & +\int_{\mathcal{I}} \widehat{\operatorname{grad}} \delta \hat{\boldsymbol{\eta}}:[\widehat{\boldsymbol{C}}: \widehat{\operatorname{grad}} \hat{\boldsymbol{z}}] \mathrm{d} S \\
& +\left[\int_{\mathcal{B}} \operatorname{grad} \delta \boldsymbol{\eta}: \boldsymbol{C} \mathrm{d} V+\int_{\mathcal{I}} \widehat{\operatorname{grad}} \delta \hat{\boldsymbol{\eta}}: \widehat{\boldsymbol{C}} \mathrm{d} S\right]: \overline{\boldsymbol{\epsilon}}=0 .
\end{aligned}
$$

The above problem is linear with respect to $z$, which can be expressed in the form [15]

$$
\boldsymbol{z}=\overline{\boldsymbol{\epsilon}}: \boldsymbol{U}, \quad \hat{\boldsymbol{z}}=\overline{\boldsymbol{\epsilon}}: \widehat{\boldsymbol{U}}, \widehat{\boldsymbol{U}}=\left.\boldsymbol{U}\right|_{\mathcal{I}},
$$

plus an arbitrary macro-quantity. $\boldsymbol{U}$ and $\widehat{\boldsymbol{U}}$ are periodic third-order tensors and they are the solutions of the auxiliary tensor equations that derive from equation (22) for arbitrary $\bar{\epsilon}$,

$$
\int_{\mathcal{B}} \operatorname{grad} \delta \boldsymbol{\eta}:[\boldsymbol{C}+\boldsymbol{C} \tilde{:} \operatorname{grad} \boldsymbol{U}] \mathrm{d} V+\int_{\mathcal{I}} \widehat{\operatorname{grad}} \delta \hat{\boldsymbol{\eta}}:[\widehat{\boldsymbol{C}}+\widehat{\boldsymbol{C}} \tilde{:} \widehat{\operatorname{grad}} \widehat{\boldsymbol{U}}] \mathrm{d} S=0 .
$$

From the definition of the macroscopic stress we have that

$$
\overline{\boldsymbol{\sigma}}=\langle\boldsymbol{\sigma}\rangle=\frac{1}{V} \int_{\mathcal{B}} \boldsymbol{\sigma} \mathrm{d} V+\frac{1}{V} \int_{\mathcal{I}} \hat{\boldsymbol{\sigma}} \mathrm{d} S=\left[\frac{1}{V} \int_{\mathcal{B}}[\boldsymbol{C}+\boldsymbol{C} \tilde{\operatorname{grad} \boldsymbol{U}}] \mathrm{d} V+\frac{1}{V} \int_{\mathcal{I}}[\widehat{\boldsymbol{C}}+\widehat{\boldsymbol{C}} \tilde{: \operatorname{grad}} \widehat{\boldsymbol{U}}] \mathrm{d} S\right]: \overline{\boldsymbol{\epsilon}},
$$

which indicates that the effective stiffness tensor with $\overline{\boldsymbol{\sigma}}=\overline{\boldsymbol{C}}: \overline{\boldsymbol{\epsilon}}$ is given by

$$
\overline{\boldsymbol{C}}=\frac{1}{V} \int_{\mathcal{B}}[\boldsymbol{C}+\boldsymbol{C} \tilde{\operatorname{grad} \boldsymbol{U}}] \mathrm{d} V+\frac{S}{V} \frac{1}{S} \int_{\mathcal{I}}[\widehat{\boldsymbol{C}}+\widehat{\boldsymbol{C}} \tilde{\operatorname{grad}} \widehat{\boldsymbol{U}}] \mathrm{d} S,
$$

where $S$ is the total area of the energetic interface. The last relation indicates that the effective stiffness tensor depends not only on the material properties of the bulk materials and the interface, but also on the ratio $S / V$. Thus in composites with micro- or nanoconstituents, where the surface-to-volume ratio is significant, the RVE size plays a role in the overall behaviour. 


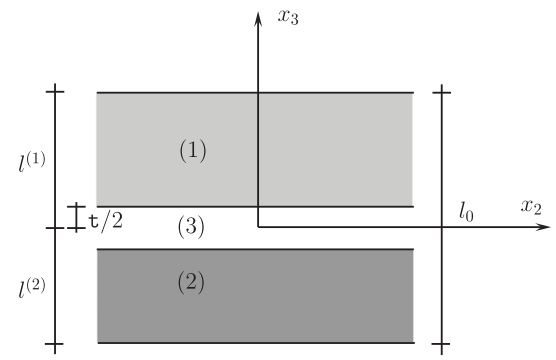

(a)

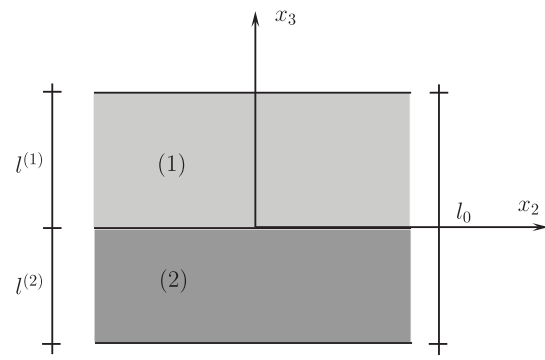

(b)

Figure 4. Multilayered composite, where a small thickness interphase layer is substituted by an energetic interface.

\section{Example: Multilayered composites with orthotropic constituents}

In order to demonstrate the theoretical analysis of the previous section, we will study the case of a multilayered material, where the solution of the RVE problem can be obtained analytically. For simplicity, in the sequel we do not distinguish between covariant and contravariant tensors, using only lower indices. We consider the case of a composite with an RVE consisting of two orthotropic materials separated in the $x_{3}$-direction by an interphase orthotropic layer with very small thickness $t$ (Figure 4). The interphase is perfectly bonded with the two materials. When $t \rightarrow 0$ the layer can be substituted by an energetic interface. The aim of this example is to identify the relation between the material properties of the interphase layer and the energetic interface which provide the same effective behaviour to the composite. In the sequel the upper indices 1 and 2 in parentheses denote bulk materials and the index 3 denotes the interphase layer. For a linear elastic orthotropic bulk material the only non-zero components of $\boldsymbol{C}$ are $C_{1111}, C_{2222}, C_{3333}, C_{1122}, C_{1133}, C_{2233}, C_{1212}, C_{1313}, C_{2323}$ and their symmetric counterparts, while for a linear elastic orthotropic energetic interface normal to the $x_{3}$-direction the only non-zero components of $\widehat{\boldsymbol{C}}$ are $\widehat{C}_{1111}, \widehat{C}_{2222}, \widehat{C}_{1122}, \widehat{C}_{1212}$ and their symmetric counterparts.

1. We first consider the energetic interface with zero thickness. Using indicial notation, the auxiliary problems (24) in both the bulk and the energetic interface read, in local form,

$$
\begin{aligned}
\frac{\partial}{\partial x_{1}}\left[C_{i 1 k l}^{(w)}+C_{i 1 m n}^{(w)} \frac{\partial U_{k l m}^{(w)}}{\partial x_{n}}\right] & +\frac{\partial}{\partial x_{2}}\left[C_{i 2 k l}^{(w)}+C_{i 2 m n}^{(w)} \frac{\partial U_{k l m}^{(w)}}{\partial x_{n}}\right] \\
& +\frac{\partial}{\partial x_{3}}\left[C_{i 3 k l}^{(w)}+C_{i 3 m n}^{(w)} \frac{\partial U_{k l m}^{(w)}}{\partial x_{n}}\right]=0 \\
\frac{\partial}{\partial x_{1}}\left[\widehat{C}_{i 1 k l}+\widehat{C}_{i 1 \alpha \beta} \frac{\partial \widehat{U}_{k l \alpha}}{\partial x_{\beta}}\right] & +\frac{\partial}{\partial x_{2}}\left[\widehat{C}_{i 2 k l}+\widehat{C}_{i 2 \alpha \beta} \frac{\partial \widehat{U}_{k l \alpha}}{\partial x_{\beta}}\right] \\
& +\llbracket C_{i 3 k l}+C_{i 3 m n} \frac{\partial U_{k l m}}{\partial x_{n}} \rrbracket=0
\end{aligned}
$$

where $w=1,2$, subjected to the continuity conditions $\widehat{\boldsymbol{U}}=\left.\boldsymbol{U}\right|_{\mathcal{I}}$. Due to the geometry of the structure, the solution depends exclusively on $x_{3}$. In such a case the interface equilibrium equations (29) lead to the classical traction continuity conditions and the equilibrium (27) reduces to a system of second-order ordinary differential equations. Solving the linear problem and using equation (25), the effective properties of the three-phase material (two bulk and one interface) are eventually obtained as

$$
\begin{aligned}
& \bar{C}_{3333}=\left[\sum_{w=1}^{2}\left[\frac{l^{(w)}}{l_{0} C_{3333}^{(w)}}\right]\right]^{-1}, \quad \bar{C}_{1133}=\bar{C}_{3333} \sum_{w=1}^{2}\left[\frac{l^{(w)} C_{1133}^{(w)}}{l_{0} C_{3333}^{(w)}}\right], \quad \bar{C}_{2233}=\bar{C}_{3333} \sum_{w=1}^{2}\left[\frac{l^{(w)} C_{2233}^{(w)}}{l_{0} C_{3333}^{(w)}}\right], \\
& \bar{C}_{1313}=\left[\sum_{w=1}^{2}\left[\frac{l^{(w)}}{l_{0} C_{1313}^{(w)}}\right]\right]^{-1}, \quad \bar{C}_{2323}=\left[\sum_{w=1}^{2}\left[\frac{l^{(w)}}{l_{0} C_{2323}^{(w)}}\right]\right]^{-1}, \quad \bar{C}_{1212}=\sum_{w=1}^{2}\left[\frac{l^{(w)}}{l_{0}} C_{1212}^{(w)}\right]+\frac{\widehat{C}_{1212}}{l_{0}},
\end{aligned}
$$




$$
\begin{aligned}
& \bar{C}_{1111}=\bar{C}_{1133} \frac{\bar{C}_{1133}}{\bar{C}_{3333}}+\sum_{w=1}^{2}\left[\frac{l^{(w)}}{l_{0}}\left[C_{1111}^{(w)}-C_{1133}^{(w)} \frac{C_{1133}^{(w)}}{C_{3333}^{(w)}}\right]\right]+\frac{\widehat{C}_{1111}}{l_{0}}, \\
& \bar{C}_{2222}=\bar{C}_{2233} \frac{\bar{C}_{2233}}{\bar{C}_{3333}}+\sum_{w=1}^{2}\left[\frac{l^{(w)}}{l_{0}}\left[C_{2222}^{(w)}-C_{2233}^{(w)} \frac{C_{2233}^{(w)}}{C_{3333}^{(w)}}\right]\right]+\frac{\widehat{C}_{2222}}{l_{0}}, \\
& \bar{C}_{1122}=\bar{C}_{1133} \frac{\bar{C}_{2233}}{\bar{C}_{3333}}+\sum_{w=1}^{2}\left[\frac{l^{(w)}}{l_{0}}\left[C_{1122}^{(w)}-C_{1133}^{(w)} \frac{C_{2233}^{(w)}}{C_{3333}^{(w)}}\right]\right]+\frac{\widehat{C}_{1122}}{l_{0}} .
\end{aligned}
$$

Computational details are given in Appendix A.

2. In the second step we consider the interphase layer with non-zero thickness $t$. The problem of different units of the mechanical properties of the layer (force per area) compared to those of the energetic interface (force per length) can be resolved by considering that the interphase layer properties are inversely proportional to $t$. Thus, the $\boldsymbol{C}^{(3)}$ interphase mechanical properties can be written in the general format $\boldsymbol{C}^{(3)}=\tilde{\boldsymbol{C}} / \mathrm{t}$. Such an approach has been followed by Duan et al. [38] in the case of isotropic energetic interfaces. The auxiliary problems (24) in local form are exactly the same as (27), with the difference that $w$ takes the value of 3 for the interphase layer. Due to the geometry of the structure, the solution depends exclusively on $x_{3}$ and the solution of the above problem provides the following effective properties:

$$
\begin{aligned}
& \bar{C}_{3333}=\left[\sum_{w=1}^{2}\left[\frac{l^{(w)}-\mathrm{t} / 2}{l_{0} C_{3333}^{(w)}}\right]+\frac{\mathrm{t}^{2}}{l_{0} \tilde{C}_{3333}}\right]^{-1}, \\
& \bar{C}_{1133}=\bar{C}_{3333}\left[\sum_{w=1}^{2}\left[\frac{\left[l^{(w)}-\mathrm{t} / 2\right] C_{1133}^{(w)}}{l_{0} C_{3333}^{(w)}}\right]+\frac{\mathrm{t} \tilde{C}_{1133}}{l_{0} \tilde{C}_{3333}}\right] \text {, } \\
& \bar{C}_{2233}=\bar{C}_{3333}\left[\sum_{w=1}^{2}\left[\frac{\left[l^{(w)}-\mathrm{t} / 2\right] C_{2233}^{(w)}}{l_{0} C_{3333}^{(w)}}\right]+\frac{\mathrm{t} \tilde{C}_{2233}}{l_{0} \tilde{C}_{3333}}\right] \text {, } \\
& \bar{C}_{1313}=\left[\sum_{w=1}^{2}\left[\frac{l^{(w)}-\mathrm{t} / 2}{l_{0} C_{1313}^{(w)}}+\frac{\mathrm{t}^{2}}{l_{0} \tilde{C}_{1313}}\right]\right]^{-1}, \\
& \bar{C}_{2323}=\left[\sum_{w=1}^{2}\left[\frac{l^{(w)}-\mathrm{t} / 2}{l_{0} C_{2323}^{(w)}}\right]+\frac{\mathrm{t}^{2}}{l_{0} \tilde{C}_{2323}}\right]^{-1}, \\
& \bar{C}_{1212}=\sum_{w=1}^{2}\left[\frac{l^{(w)}-\mathrm{t} / 2}{l_{0}} C_{1212}^{(w)}\right]+\frac{\tilde{C}_{1212}}{l_{0}}, \\
& \bar{C}_{1111}=\bar{C}_{1133} \frac{\bar{C}_{1133}}{\bar{C}_{3333}}+\sum_{w=1}^{2}\left[\frac{l^{(w)}-\mathrm{t} / 2}{l_{0}}\left[C_{1111}^{(w)}-C_{1133}^{(w)} \frac{C_{1133}^{(w)}}{C_{3333}^{(w)}}\right]\right]+\frac{1}{l_{0}}\left[\tilde{C}_{1111}-\tilde{C}_{1133} \frac{\tilde{C}_{1133}}{\tilde{C}_{3333}}\right], \\
& \bar{C}_{2222}=\bar{C}_{2233} \frac{\bar{C}_{2233}}{\bar{C}_{3333}}+\sum_{w=1}^{2}\left[\frac{l^{(w)}-\mathrm{t} / 2}{l_{0}}\left[C_{2222}^{(w)}-C_{2233}^{(w)} \frac{C_{2233}^{(w)}}{C_{3333}^{(w)}}\right]\right]+\frac{1}{l_{0}}\left[\tilde{C}_{2222}-\tilde{C}_{2233} \frac{\tilde{C}_{2233}}{\tilde{C}_{3333}}\right] \text {, } \\
& \bar{C}_{1122}=\bar{C}_{1133} \frac{\bar{C}_{2233}}{\bar{C}_{3333}}+\sum_{w=1}^{2}\left[\frac{l^{(w)}-\mathrm{t} / 2}{l_{0}}\left[C_{1122}^{(w)}-C_{1133}^{(w)} \frac{C_{2233}^{(w)}}{C_{3333}^{(w)}}\right]\right]+\frac{1}{l_{0}}\left[\tilde{C}_{1122}-\tilde{C}_{1133} \frac{\tilde{C}_{2233}}{\tilde{C}_{3333}}\right] \text {. }
\end{aligned}
$$

Computational details are given in Appendix A.

Comparing the results between the two cases we observe that for $\mathrm{t} \rightarrow 0$ : 
a) They render the same effective components $\bar{C}_{3333}, \bar{C}_{1133}, \bar{C}_{2233}, \bar{C}_{1323}, \bar{C}_{2323}$;

b) They render the same effective component $\bar{C}_{1212}$ only if $\widehat{C}_{1212}=\tilde{C}_{1212}$;

c) They render the same effective component $\bar{C}_{1111}$ only if

$$
\widehat{C}_{1111}=\tilde{C}_{1111}-\tilde{C}_{1133} \frac{\tilde{C}_{1133}}{\tilde{C}_{3333}} ;
$$

d) They render the same effective component $\bar{C}_{2222}$ only if

$$
\widehat{C}_{2222}=\tilde{C}_{2222}-\tilde{C}_{2233} \frac{\tilde{C}_{2233}}{\tilde{C}_{3333}} ;
$$

e) They render the same effective component $\bar{C}_{1122}$ only if

$$
\widehat{C}_{1122}=\tilde{C}_{1122}-\tilde{C}_{1133} \frac{\tilde{C}_{2233}}{\tilde{C}_{3333}} .
$$

From the above analysis we conclude that a material layer with very small thickness $t$ and elasticity tensor $\boldsymbol{C}^{(3)}$ can be substituted by an energetic interface with properties

$$
\begin{array}{ll}
\widehat{C}_{1111}=\left[C_{111}^{(3)}-C_{1133}^{(3)} \frac{C_{1133}^{(3)}}{C_{3333}^{(3)}}\right] \mathrm{t}, & \widehat{C}_{1122}=\left[C_{1122}^{(3)}-C_{1133}^{(3)} \frac{C_{2233}^{(3)}}{C_{3333}^{(3)}}\right] \mathrm{t}, \\
\widehat{C}_{2222}=\left[C_{2222}^{(3)}-C_{2233}^{(3)} \frac{C_{2233}^{(3)}}{C_{3333}^{(3)}}\right] \mathrm{t}, & \widehat{C}_{1212}=C_{1212}^{(3)} \mathrm{t},
\end{array}
$$

and provide the same effective behaviour for a multilayered composite. In the case of isotropic material constituents and an isotropic energetic interface the above formulae are reduced to those obtained by Wang et al. [39] and Duan et al. [38] for composites with spherical particles or cylindrical fibres.

\section{Summary and conclusions}

The paper presents a homogenization framework that accounts for size effects at the micro- or nanoscale through the introduction of material interfaces based on surface elasticity theory. The connection between the scales requires integrals over the RVE in the bulk and on the interface, while the microscopic deformation in terms of the macroscopic strain tensor is defined through a Hill-type averaging condition that links the two scales. The definition of average (macroscopic) stress given by Benveniste and Miloh [37] for composites with imperfect interfaces needs proper modification when open energetic interfaces are present. The overall effective properties in the case of composites with elastic components and elastic material interfaces depend not only on the material properties and volume fractions, but also on the surface-to-volume ratio. A particular example of a multilayered orthotropic composite demonstrates that a very thin interphase layer can be represented by a 2-D material interface after proper transformation of the actual 3-D elasticity tensor.

\section{Funding}

The support of this work by the ERC Advanced Grant MOCOPOLY is gratefully acknowledged.

\section{Conflict of interest}

None declared.

\section{References}

[1] Gibbs, JW. The scientific papers of JW Gibbs. Volume 1. New York: Dover Publications, 1961.

[2] Gurtin, ME, and Murdoch, AI. A continuum theory of elastic material surfaces. Arch Rat Mech Anal 1975; 57(4): $291-323$. 
[3] Moeckel, GP. Thermodynamics of an interface. Arch Rat Mech Anal 1975; 57: 255-280.

[4] Daher, N, and Maugin, GA. The method of virtual power in continuum mechanics application to media presenting singular surfaces and interfaces. Acta Mech 1986; 60(3-4): 217-240.

[5] Dell'Isola, F, and Romano, A. On the derivation of thermomechanical balance equations for continuous systems with a nonmaterial interface. Int J Eng Sci 1987; 25(11-12): 1459-1468.

[6] Steinmann, P. On boundary potential energies in deformational and configurational mechanics. J Mech Phys Solids 2008; 56(3): 772-800.

[7] Hill, R. Elastic properties of reinforced solids: Some theoretical principles. J Mech Phys Solids 1963; 11: 357-372.

[8] Hill, R, and Rice, J. Constitutive analysis of elastic-plastic crystals at arbitrary strain. J Mech Phys Solids 1972; $20(6)$ : $401-413$.

[9] Charalambakis, N. Homogenization techniques and micromechanics. A survey and perspectives. Appl Mech Rev 2010; 63(3): 030803.

[10] Kanouté, P, Boso, DP, Chaboche, JL, et al. Multiscale methods for composites: A review. Arch Comput Meth Eng 2009; 16: 31-75.

[11] Bensoussan, A, Lions, J, and Papanicolaou, G. Asymptotic methods for periodic structures. Amsterdam: North Holland, 1978.

[12] Hashin, Z and Shtrikman, S. A variational approach to the theory of the elastic behaviour of multiphase materials. J Mech Phys Solids 1963; 11(2): 127-140.

[13] Miehe, C, and Koch, A. Computational micro-to-macro transitions of discretized microstructures undergoing small strains. Arch Appl Mech 2002; 72(4-5): 300-317.

[14] Mura, T. Micromechanics of defects in solids. Boston: Martinus Nijhoff Publishers, 1987.

[15] Sanchez-Palencia, E. Non-homogeneous media and vibration theory (Lecture Notes in Physics, vol. 127). Berlin: Springer-Verlag, 1978.

[16] Suquet, PM. Elements of homogenization for inelastic solid mechanics (Lecture Notes in Physics, vol. 272). Berlin: SpringerVerlag, 1987, 193-278.

[17] Hill, R. On constitutive macro-variables for heterogeneous solids at finite strain. Proc R Soc Lond Ser A 1972; 326(1565): 131-147.

[18] Hirschberger, CB, Sukumar, N, and Steinmann, P. Computational homogenization of material layers with micromorphic mesostructure. Philos Mag 2008; 88(30): 3603-3631.

[19] McBride, A, Mergheim, J, Javili, A, et al. Micro-to-macro transitions for heterogeneous material layers accounting for in-plane stretch. J Mech Phys Solids 2012; 60(6): 1221-1239.

[20] Miehe, C. Computational micro-to-macro transitions for discretized micro-structures of heterogeneous materials at finite strains based on the minimization of averaged incremental energy. Comput Meth Appl Mech Eng 2003; 192(5-6): 559-591.

[21] Miehe, C, Schröder, J, and Schotte, J. Computational homogenization analysis in finite plasticity simulation of texture development in polycrystalline materials. Comput Meth Appl Mech Eng 1999; 171(3-4): 387-418.

[22] Nemat-Nasser, S. Averaging theorems in finite deformation plasticity. Mech Mater 1999; 31: 493-523.

[23] Ricker, S, Mergheim, J, Steinmann, P, et al. A comparison of different approaches in the multi-scale computation of configurational forces. Int J Fract 2010; 166: 203-214.

[24] Javili, A, and Steinmann, P. A finite element framework for continua with boundary energies. Part I: The two-dimensional case. Comput Meth Appl Mech Eng 2009; 198(27-29): 2198-2208.

[25] Javili, A, and Steinmann, P. A finite element framework for continua with boundary energies. Part II: The three-dimensional case. Comput Meth Appl Mech Eng 2010; 199(9-12): 755-765.

[26] Kaptay, G. Classification and general derivation of interfacial forces, acting on phases, situated in the bulk, or at the interface of other phases. J Mater Sci 2005; 40: 2125-2131.

[27] Wei, GW, Shouwen, Y, and Ganyun, H. Finite element characterization of the size-dependent mechanical behaviour in nanosystems. Nanotech 2006; 17(4): 1118-1122.

[28] Duan, HL, Wang, J, Huang, ZP, et al. Size-dependent effective elastic constants of solids containing nano-inhomogeneities with interface stress. J Mech Phys Solids 2005; 53(7): 1574-1596.

[29] Duan, HL, Wang, J, Karihaloo, BL, et al. Nanoporous materials can be made stiffer than non-porous counterparts by surface modification. Acta Mater 2006; 54: 2983-2990.

[30] Sharma, P, and Ganti, S. Size-dependent Eshelby's tensor for embedded nano-inclusions incorporating surface/interface energies. J Appl Mech 2004; 71: 663-671.

[31] Gurtin, ME, Fried, E, and Anand, L. The mechanics and thermodynamics of continua. Cambridge: Cambridge University Press, 2009.

[32] Marsden, JE and Hughes, TJR. Mathematical foundations of elasticity. New York: Dover Publications, 1994.

[33] Maugin, GA. Configurational forces: Thermomechanics, physics, mathematics, and numerics. Boca Raton, FL: CRC Press, 2010.

[34] Ogden, RW. Non-linear elastic deformations. New York: Dover Publications, 1997.

[35] Truesdell, C, and Noll, W. The non-linear field theories of mechanics. 3rd edn. Berlin: Springer-Verlag, 2004.

[36] Javili, A, McBride, A and Steinmann, P. Thermomechanics of solids with lower-dimensional energetics: On the importance of surface, interface and curve structures at the nanoscale. A unifying review. Appl Mech Rev 2013; 65: 010802.

[37] Benveniste, Y, and Miloh, T. Imperfect soft and stiff interfaces in two-dimensional elasticity. Mech Mater 2001; 33(6): 309-323. 
[38] Duan, HL, Yi, X, Huang, ZP, et al. A unified scheme for prediction of effective moduli of multiphase composites with interface effects. Part I: Theoretical framework. Mech Mater 2007; 39(1): 81-93.

[39] Wang, J, Duan, H, Zhang, Z, et al. An anti-interpenetration model and connections between interphase and interface models in particle-reinforced composites. Int J Mech Sci 2005; 47: 701-708.

\section{Appendix A. Analytical solution for multilayered composites}

In the example in Section 5 we examine two cases:

1. Two bulk material layers $(w=1,2)$ and one energetic interface are considered. Due to the nature of the problem, the solution of the system of equations (29), subject to interface conditions (27) and continuity of displacements, depends exclusively on $x_{3}$. Considering only the non-zero terms of $\boldsymbol{C}$, we can re-express the problem as

$$
\frac{\partial}{\partial x_{3}}\left[C_{13 k l}^{(w)}+C_{1313}^{(w)} \frac{\partial U_{k l 1}^{(w)}}{\partial x_{3}}\right]=0, \quad \frac{\partial}{\partial x_{3}}\left[C_{23 k l}^{(w)}+C_{2323}^{(w)} \frac{\partial U_{k l 2}^{(w)}}{\partial x_{3}}\right]=0, \quad \frac{\partial}{\partial x_{3}}\left[C_{33 k l}^{(w)}+C_{3333}^{(w)} \frac{\partial U_{k l 3}^{(w)}}{\partial x_{3}}\right]=0,
$$

subject to the periodicity conditions

$$
\left.U_{k l 1}^{(1)}\right|_{x_{3}=l^{(1)}}=\left.U_{k l 1}^{(2)}\right|_{x_{3}=-l^{(2)}},\left.\quad U_{k l 2}^{(1)}\right|_{x_{3}=l^{(1)}}=\left.U_{k l 2}^{(2)}\right|_{x_{3}=-l^{(2)}},\left.\quad U_{k l 3}^{(1)}\right|_{x_{3}=l^{(1)}}=\left.U_{k l 3}^{(2)}\right|_{x_{3}=-l^{(2)}},
$$

and the continuity conditions on $\mathcal{I}$,

$$
\begin{aligned}
& {\left.\left[C_{13 k l}^{(1)}+C_{1313}^{(1)} \frac{\partial U_{k l 1}^{(1)}}{\partial x_{3}}\right]\right|_{x_{3}=0}=\left.\left[C_{13 k l}^{(2)}+C_{1313}^{(2)} \frac{\partial U_{k l 1}^{(2)}}{\partial x_{3}}\right]\right|_{x_{3}=0}, } \\
& {\left.\left[C_{23 k l}^{(1)}+C_{2323}^{(1)} \frac{\partial U_{k l 2}^{(1)}}{\partial x_{3}}\right]\right|_{x_{3}=0}=\left.\left[C_{23 k l}^{(2)}+C_{2323}^{(2)} \frac{\partial U_{k l 2}^{(2)}}{\partial x_{3}}\right]\right|_{x_{3}=0}, } \\
& {\left.\left[C_{33 k l}^{(1)}+C_{3333}^{(1)} \frac{\partial U_{k l 3}^{(1)}}{\partial x_{3}}\right]\right|_{x_{3}=0}=\left.\left[C_{33 k l}^{(2)}+C_{3333}^{(2)} \frac{\partial U_{k l 3}^{(2)}}{\partial x_{3}}\right]\right|_{x_{3}=0}, } \\
&\left.U_{k l 1}^{(1)}\right|_{x_{3}=0}=\left.U_{k l 1}^{(2)}\right|_{x_{3}=0},\left.\quad U_{k l 2}^{(1)}\right|_{x_{3}=0}=\left.U_{k l 2}^{(2)}\right|_{x_{3}=0},\left.\quad U_{k l 3}^{(1)}\right|_{x_{3}=0}=\left.U_{k l 3}^{(2)}\right|_{x_{3}=0} .
\end{aligned}
$$

The indices $k$ and $l$ take the values 1,2 or 3 . The first three continuity conditions of (33) allow integrating equations (31) and obtain

$$
C_{13 k l}^{(w)}+C_{1313}^{(w)} \frac{\partial U_{k l 1}^{(w)}}{\partial x_{3}}=\omega_{k l 1}, \quad C_{23 k l}^{(w)}+C_{2323}^{(w)} \frac{\partial U_{k l 2}^{(w)}}{\partial x_{3}}=\omega_{k l 2}, \quad C_{33 k l}^{(w)}+C_{3333}^{(w)} \frac{\partial U_{k l 3}^{(w)}}{\partial x_{3}}=\omega_{k l 3},
$$

where $\omega_{k l 1}, \omega_{k l 2}$ and $\omega_{k l 3}$ are constants independent of the material layer. Integration of the last relations leads to

$$
U_{k l 1}^{(w)}=\frac{\omega_{k l 1}-C_{13 k l}^{(w)}}{C_{1313}^{(w)}} x_{3}+d_{k l 1}^{(w)}, \quad U_{k l 2}^{(w)}=\frac{\omega_{k l 2}-C_{23 k l}^{(w)}}{C_{2323}^{(w)}} x_{3}+d_{k l 2}^{(w)}, \quad U_{k l 3}^{(w)}=\frac{\omega_{k l 3}-C_{33 k l}^{(w)}}{C_{3333}^{(w)}} x_{3}+d_{k l 3}^{(w)},
$$

where $d_{k l 1}^{(w)}, d_{k l 2}^{(w)}$ and $d_{k l 3}^{(w)}$ are constants with different values at each material layer. From the last three continuity conditions of (33) and the periodicity conditions (32) we compute the constants $\omega_{k l 1}, \omega_{k l 2}$ and $\omega_{k l 3}$,

$$
\omega_{k l 1}=\left\langle\frac{C_{13 k l}^{(w)}}{C_{1313}^{(w)}}\right\rangle\left\langle\frac{1}{C_{1313}^{(w)}}\right\rangle^{-1}, \quad \omega_{k l 2}=\left\langle\frac{C_{23 k l}^{(w)}}{C_{2323}^{(w)}}\right\rangle\left\langle\frac{1}{C_{2323}^{(w)}}\right\rangle^{-1}, \quad \omega_{k l 3}=\left\langle\frac{C_{33 k l}^{(w)}}{C_{3333}^{(w)}}\right\rangle\left\langle\frac{1}{C_{3333}^{(w)}}\right\rangle^{-1},
$$


where the symbol $\langle\{\bullet\}\rangle$ for constant terms inside each bulk material renders the usual rule of mixtures. The equations (34), (36) and (25) allow expression of the non-zero components of the symmetric effective stiffness tensor as

$$
\begin{gathered}
\bar{C}_{3333}=\omega_{333}=\left\langle\frac{1}{C_{3333}^{(w)}}\right\rangle^{-1}, \bar{C}_{1133}=\bar{C}_{3311}=\omega_{113}=\bar{C}_{3333}\left\langle\frac{C_{1133}^{(w)}}{C_{3333}^{(w)}}\right\rangle, \\
\bar{C}_{2233}=\bar{C}_{3322}=\omega_{223}=\bar{C}_{3333}\left\langle\frac{C_{2233}^{(w)}}{C_{3333}^{(w)}}\right\rangle, \bar{C}_{1313}=\omega_{131}=\left\langle\frac{1}{C_{1313}^{(w)}}\right\rangle^{-}, \\
\bar{C}_{2323}=\omega_{232}=\left\langle\frac{1}{C_{2323}^{(w)}}\right\rangle, \bar{C}_{1212}=\left\langle C_{1212}\right\rangle+\frac{\widehat{C}_{1212}}{l_{0}}, \\
\bar{C}_{1111}=\left\langle\begin{array}{l}
\left.C_{111}^{(w)}+C_{1133}^{(w)} \frac{\partial U_{113}^{(w)}}{\partial x_{3}}\right\rangle+\frac{\widehat{C}_{1111}}{l_{0}}=\bar{C}_{1133} \frac{\bar{C}_{1133}}{\bar{C}_{3333}}+\left\langle C_{1111}^{(w)}-C_{1133}^{(w)} \frac{C_{1133}^{(w)}}{C_{3333}^{(w)}}\right\rangle+\frac{\widehat{C}_{1111}}{l_{0}}, \\
\left.\widehat{C}_{2222}^{(w)}+C_{2222}^{(w)}+C_{2233} \frac{\partial U_{223}^{(w)}}{\partial x_{3}}\right\rangle+\frac{\widehat{C}_{2222}}{l_{0}}=\bar{C}_{2233} \frac{\widehat{C}_{2233}}{\bar{C}_{3333}}+\left\langle C_{2222}^{(w)}-C_{2233}^{(w)} \frac{C_{2233}^{(w)}}{C_{3333}^{(w)}}\right\rangle+\frac{C_{2222}}{l_{0}}, \\
\bar{C}_{2211}=\bar{C}_{1122}=\left\langle\widehat{C}_{1122}^{(w)}+C_{1133}^{(w)} \frac{\partial U_{223}^{(w)}}{\partial x_{3}}\right\rangle+\frac{\widehat{C}_{1122}}{l_{0}}=\bar{C}_{1133} \frac{\bar{C}_{2233}}{\bar{C}_{3333}}+\left\langle C_{1122}^{(w)}-C_{1133}^{(w)} \frac{C_{2233}}{C_{3333}^{(w)}}\right\rangle+\frac{\widehat{C}_{1122}}{l_{0}} .
\end{array}\right.
\end{gathered}
$$

2. Three bulk material layers $(w=1,2,3)$ are considered, without any energetic interface. Then the system (31) and the periodicity conditions (32) are the same as with the previous case, while the continuity conditions are written as

$$
\begin{aligned}
& \begin{array}{l}
{\left.\left[C_{13 k l}^{(1)}+C_{1313}^{(1)} \frac{\partial U_{k l 1}^{(1)}}{\partial x_{3}}\right]\right|_{x_{3}=\mathrm{t} / 2}=\left.\left[C_{13 k l}^{(3)}+C_{1313}^{(3)} \frac{\partial U_{k l 1}^{(3)}}{\partial x_{3}}\right]\right|_{x_{3}=\mathrm{t} / 2},} \\
{\left.\left[C_{23 k l}^{(1)}+C_{2323}^{(1)} \frac{\partial U_{k l 2}^{(1)}}{\partial x_{3}}\right]\right|_{x_{3}=\mathrm{t} / 2}=\left.\left[C_{23 k l}^{(3)}+C_{2323}^{(3)} \frac{\partial U_{k l 2}^{(3)}}{\partial x_{3}}\right]\right|_{x_{3}=\mathrm{t} / 2},}
\end{array} \\
& {\left.\left[C_{33 k l}^{(1)}+C_{3333}^{(1)} \frac{\partial U_{k l 3}^{(1)}}{\partial x_{3}}\right]\right|_{x_{3}=\mathrm{t} / 2} ^{x_{3}=\mathrm{t} / 2}=\left.\left[C_{33 k l}^{(3)}+C_{3333}^{(3)} \frac{\partial U_{k l 3}^{(3)}}{\partial x_{3}}\right]\right|_{x_{3}=\mathrm{t} / 2} ^{x_{3}=\mathrm{t} / 2},} \\
& {\left.\left[C_{13 k l}^{(2)}+C_{1313}^{(2)} \frac{\partial U_{k l 1}^{(2)}}{\partial x_{3}}\right]\right|_{x_{3}=-\mathrm{t} / 2} ^{x_{3}=\mathrm{t} / 2}=\left.\left[C_{13 k l}^{(3)}+C_{1313}^{(3)} \frac{\partial U_{k l 1}^{(3)}}{\partial x_{3}}\right]\right|_{x_{3}=-\mathrm{t} / 2} ^{x_{3}=\mathrm{t} / 2},} \\
& {\left.\left[C_{23 k l}^{(2)}+C_{2323}^{(2)} \frac{\partial U_{k l 2}^{(2)}}{\partial x_{3}}\right]\right|_{x_{3}=-\mathrm{t} / 2} ^{x_{3}=-\mathrm{t} / 2}=\left.\left[C_{23 k l}^{(3)}+C_{2323}^{(3)} \frac{\partial U_{k l 2}^{(3)}}{\partial x_{3}}\right]\right|_{x_{3}=-\mathrm{t} / 2} ^{x_{3}=-\mathrm{t} / 2},} \\
& {\left.\left[C_{33 k l}^{(2)}+C_{3333}^{(2)} \frac{\partial U_{k l 3}^{(2)}}{\partial x_{3}}\right]\right|_{x_{3}=-\mathrm{t} / 2} ^{x_{3}=-\mathrm{t} / 2}=\left.\left[C_{33 k l}^{(3)}+C_{3333}^{(3)} \frac{\partial U_{k l 3}^{(3)}}{\partial x_{3}}\right]\right|_{x_{3}=-\mathrm{t} / 2} \text {, }} \\
& \left.U_{k l 1}^{(1)}\right|_{x_{3}=\mathrm{t} / 2}=\left.U_{k l 1}^{(3)}\right|_{x_{3}=\mathrm{t} / 2},\left.\quad U_{k l 2}^{(1)}\right|_{x_{3}=\mathrm{t} / 2}=\left.U_{k l 2}^{(3)}\right|_{x_{3}=\mathrm{t} / 2},\left.\quad U_{k l 3}^{(1)}\right|_{x_{3}=\mathrm{t} / 2}=\left.U_{k l 3}^{(3)}\right|_{x_{3}=\mathrm{t} / 2}, \\
& \left.U_{k l 1}^{(2)}\right|_{x_{3}=-\mathrm{t} / 2}=\left.U_{k l 1}^{(3)}\right|_{x_{3}=-\mathrm{t} / 2},\left.\quad U_{k l 2}^{(2)}\right|_{x_{3}=-\mathrm{t} / 2} ^{x_{3}=\mathrm{t} / \mathrm{t} / 2}=\left.U_{k l 2}^{(3)}\right|_{x_{3}=-\mathrm{t} / 2} ^{x_{3}=\mathrm{t} / 2},\left.\quad U_{k l 3}^{(2)}\right|_{x_{3}=-\mathrm{t} / 2}=\left.U_{k l 3}^{(3)}\right|_{x_{3}=-\mathrm{t} / 2} .
\end{aligned}
$$

The solution of this problem has the same form as with the previous case, that is, equations (35), and the unknown constants $\omega_{k l 1}, \omega_{k l 2}$ and $\omega_{k l 3}$ are computed by the similar relations (36), with the difference that in the averages one should consider the contribution of the third layer. Thus the effective properties are given by equations (37), taking into account that $\widehat{\boldsymbol{C}}=0$. 